\title{
Recurrent Dieulafoy's disease with surgical management: diagnosis by endoscopic ultrasonography
}

\author{
D. Martínez Ares, J. Souto Ruzo, J. Yáñez López, P. Alonso Aguirre, C. Gómez Mata², L. Valbuena \\ Ruvira $^{1}$ and J. L. Vázquez Iglesias
}

Service of Digestive Diseases, ${ }^{1}$ Pathology and ${ }^{2}$ General Surgery. Complejo Hospitalario Universitario Juan Canalejo. A Coruña, Spain

\begin{abstract}
Dieulafoy's disease is an uncommon but potentially significant cause of gastrointestinal bleeding caused by a large-caliber arterial vessel in the submucosa, which causes erosion and debilitation of the surrounding mucosa and may lead to massive gastrointestinal bleeding. Since endoscopy may prove insufficient, echoendoscopy may help in the diagnosis of this condition. Echoendoscopy may also help improve endoscopic management using mechanical techniques (hemoclips or band ligation) or a combination of thermal techniques and injection sclerotherapy, since this allows an accurate localization of the submucosal vessel.

We present a case illustrating this approach by endoscopic ultrasonography, and describe the morphological substrate of this condition.
\end{abstract}

Key words: Dieulafoy's disease. Endoscopic ultrasonography. Surgical management. Endoscopic management.

Martínez Ares D, Souto Ruzo J, Yáñez López J, Alonso Aguirre P, Gómez Mata C, Valbuena Ruvira L, Vázquez Iglesias JL. Recurrent Dieulafoy's disease with surgical management: diagnosis by endoscopic ultrasonography. Rev Esp Enferm Dig 2004; 96: 138-142.

\section{INTRODUCTION}

Dieulafoy's disease is an uncommon cause of gastrointestinal bleeding (1), which accounts for approximately $1 \%$ of all non-variceal upper gastrointestinal bleeding episodes (2). Most are cases of peptic ulcer (3). Nevertheless, the actual incidence of this disease is not

Recibido: 23-04-03.

Aceptado: 04-09-03.

Correspondencia: David Martínez Ares. C/ Emilio González López, 11, $7^{\circ}$ B. 15011 A Coruña. e-mail: dmartinezares@sepd.es accurately known, since misdiagnosis may occur in some cases (4). Most of these lesions are located in the stomach (1), although they may also appear in other parts of the gastrointestinal tract such as the duodenum, jejunum or rectum. Although fairly uncommon, this entity may be potentially severe and even have a fatal outcome.

It is also known as "caliber-persistent artery", and consists of a large caliber artery which protrudes through the submucosa and contacts the mucosa (5). Minute ruptures in this usually atrophied mucosa (6) lead to rupture of the vessel and cause subsequent bleeding. This bleeding may be recurrent or single. Diagnosis is difficult when no active bleeding is observed, since no apparent lesion in the mucosa can be seen (5). Initial treatment usually consists of endoscopic management: injection techniques combined with another modality of endoscopic hemostasis: thermocoagulation or mechanical methods (hemoclipping or band ligation). If the bleeding cannot be stopped by these techniques, a surgical resection of the affected segment is generally indicated.

\section{CASE REPORT}

We present the case of a 39-year-old male with a history of two previous hospital admissions 9 and 4 years before, both for upper gastrointestinal bleeding secondary to Dieulafoy's disease, one of which required surgery. The patient did not report previous alcohol abuse, smoking or other unhealthy habits. There was no history of NSAID or other drugs ingestion.

The patient presented with anemia and melenic stools at admission. Biochemistry revealed severe anemia with a hematocrit of $25 \%(38-50)$ and hemoglobin $7.5 \mathrm{~g} / \mathrm{dl}$ (13.5-18). An emergency upper endoscopy showed a protruding lesion with preserved mucosa at the junction of the body and fundus, and no active bleeding at that time, a picture that apparently corresponded to the Dieulafoy's 




Fig. 1.- An elevated lesion with no active bleeding is identified at the junction of the gastric body and fundus, apparently corresponding to a Dieulafoy's lesion. A minute bleeding spot may be seen at the site of sclerosant injection

A nivel de la unión de cuerpo y fundus gástrico se identifica lesión sobreelevada sin sangrado activo, que parecía corresponder a una lesión de Dieulafoy; en la imagen se aprecia un mínimo punto de sangrado que corresponde al lugar de la inyección de sustancia esclerosante.

disease (Fig. 1). Endoscopic ultrasonography revealed the presence of a hypoechogenic tubular structure which corresponded to an arterial vessel with an abnormally large caliber and an ulceration in its apex in contact with the mucosa, which suggested the diagnose of Dieulafoy's disease (Fig. 2). An endoscopic sclerotherapy was performed and two hemoclips were placed on the lesion. Recurrent bleeding with hematemesis, rectal bleeding and hemodynamic instability developed in the days following this treatment, requiring the transfusion of six concentrates of red blood cells. Surgical management was then decided upon, and consisted of partial gastrectomy. The pathological study revealed the presence of an aberrant dilated submucosal vessel, erosion of the overlying mucosa, luminal thrombosis, and areas of peripheral bleeding (Fig. 3). The subsequent recovery of the patient was uneventful, and bleeding has not recurred.

\section{DISCUSSION}

The diagnosis of Dieulafoy's disease is not always easy, especially when no active bleeding is observed during endoscopy. For this reason, repeat examinations may be required. Norton et al. published in 1999 a series of 90 cases in which a mean of 1.9 explorations were required before a diagnosis was reached (7). In our study, the diagnosis appeared in the form of a nodular lesion with active bleeding, suggestive of Dieulafoy's lesion. Nevertheless, this entity may have a presentation not allowing diagnosis when there is no active bleeding, or it may have a

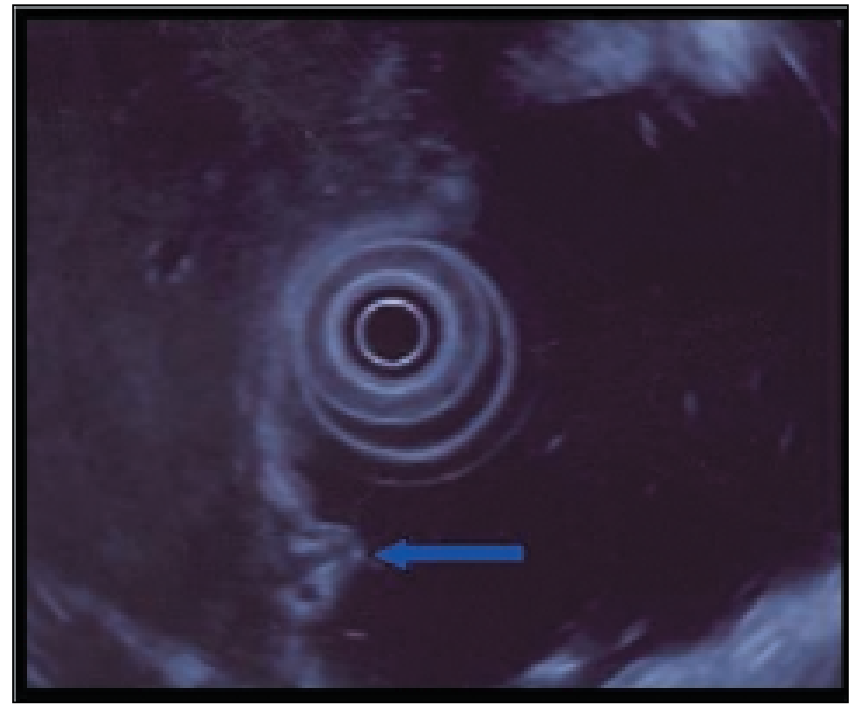

Fig. 2.- Echoendoscopy reveals the presence of an hypoechogenic tubular structure that corresponds to an arterial vessel with an abnormally large caliber and an ulceration in its apex in contact with the mucosa, which suggests the presence of a case of Dieulafoy's disease.

La ecoendoscopia muestra una estructura tubular hipoecogénica que corresponde al vaso arterial con calibre anormlamente grande en contacto con la mucosa, ulcerado en el vértice, sugestiva de enfermedad de Dieulafoy.

form similar to that of gastrointestinal varices (8). When the diagnosis is difficult to reach, endoscopic ultrasonography and Doppler imaging may help locate the largecaliber arterial vessel in contact with the mucosa, which is characteristic of this lesion $(9,10)$. In our case, this is illustrated by the images provided by echoendoscopy.

The initial management of Dieulafoy's disease should be endoscopic. Hemostasis may be equally achieved by mechanical and thermal methods, whether these are combined with injection sclerotherapy or not (1). However, endoscopic sclerosis cannot be considered the only therapeutic approach acceptable, since it does not offer the efficacy provided by the combination of sclerosis and thermal methods (2), or by mechanical treatments (11). As for mechanical options, both elastic band ligation $(12,13)$ and hemoclipping $(11,14)$ have proven to be safe, efficient and cost-effective techniques. These techniques may be combined with Doppler imaging and ultrasonography in order to better locate the vessel, thus allowing better outcomes especially in cases where the lesion is not apparent $(15,16)$. In our case, endoscopic ultrasonography confirmed the diagnosis and was of help for the placement of two hemoclips. However, despite an apparently correct placement of hemoclips and the performance of an endoscopic sclerosing of the lesion, recurrent bleeding developed, and so surgical management was performed as the definitive solution.

In conclusion, Dieulafoy's disease is an uncommon condition that is sometimes difficult to diagnose. In these cases, endoscopic ultrasonography may be helpful both to reach a diagnosis and perform a more precise endosco- 


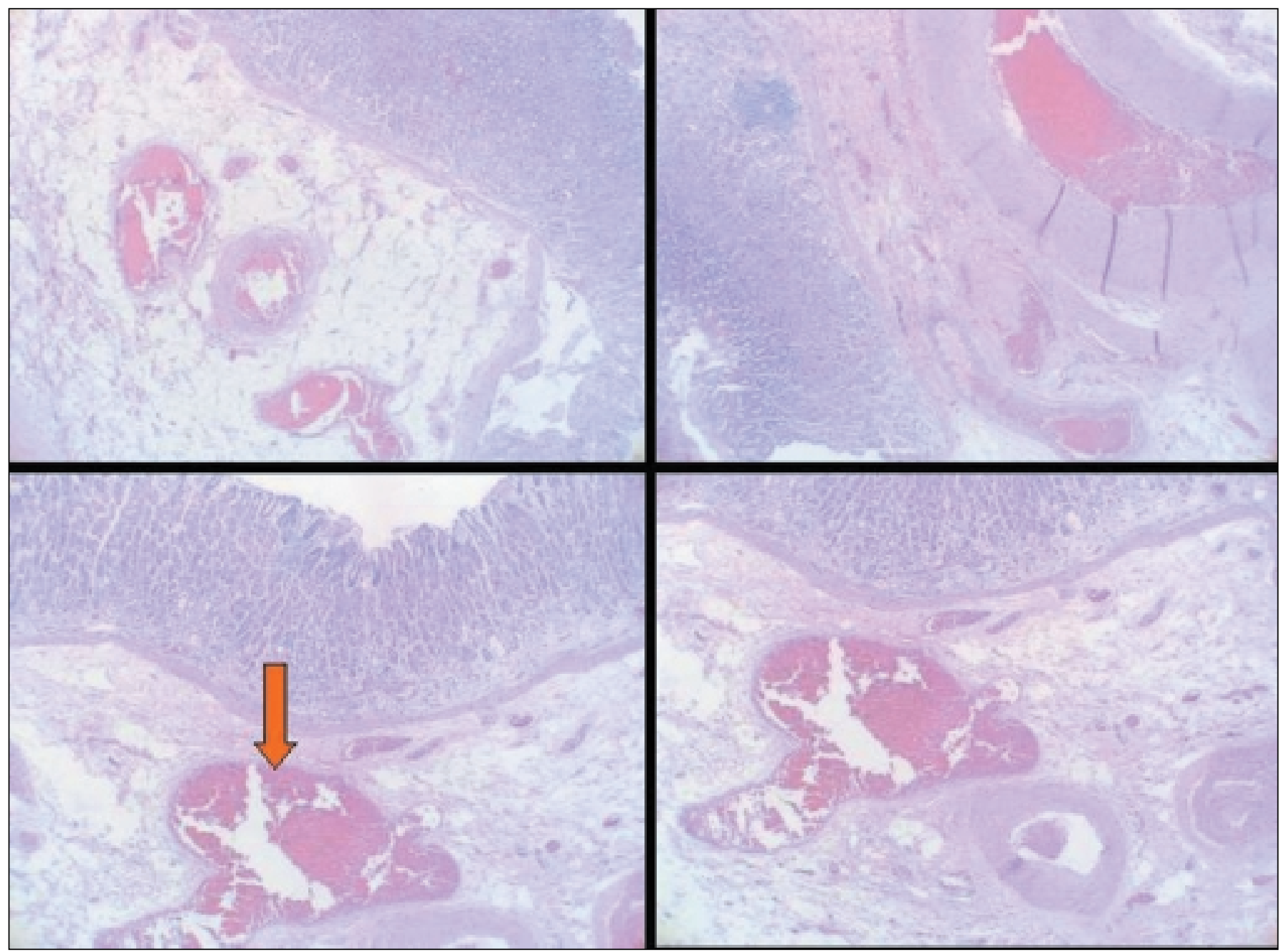

Fig. 3.- A submucosal aneurysmal vessel identified in the surgical specimen. There is erosion of the overlying mucosa, thrombosis of the lumen, and peripheral bleeding.

En la pieza quirúrgica se identifica una vaso aneurismático submucoso con erosión de la mucosa suprayacente, trombosis de la luz y zonas de hemorragia periférica.

pic approach. Surgical treatment is generally the option of choice when endoscopy is unsuccessful and when there is recurrent bleeding.

\section{REFERENCES}

1. Mumtaz R, Shaukat M, Ramírez FC. Outcomes of endoscopic treatment of gastroduodenal Dieulafoy's lesion with rubber band ligation and thermal/injection therapy. J Clin Gastroenterol 2003; 36 (4): 3104.

2. Kasapidis P, Georgopoulos P, Delis V, Balatsos V, Konstantinidis A, Skandalis N. Endoscopic management and long-term follow-up of Dieulafoy's lesions in the upper GI tract. Gastrointest Endosc 2002; 55 (4): 527-31.

3. Palmer KR. Ulcers and nonvariceal bleeding. Endoscopy 2000; 32 (2): 118-23.

4. Schmulewitz N, Baillie J. Dieulafoy lesions: a review of 6 years of experience at a tertiary referral center. Am J Gastroenterol 2001; 96 (6): 1688-94.
5. Vázquez Iglesias, JL. Alteraciones Vasculares. En: Endoscopia Digestiva Alta. Volumen I. Galcia Editorial, SA, 1992: 228-9.

6. Owaki T, Kusano C, Ojiro M, Aikou T. Massive bleeding from Dieulafoy's lesion of the small intestine in a child - therapy for the bleeding from gastrointestinal tract out of endoscopic observation. Dig Surg 2002; 19 (4): 321-4.

7. Norton ID, Petersen BT, Sorbi D, Balm RK, Alexander GL, Gostout CJ. Management and long-term prognosis of Dieulafoy lesion. Gastrointest Endosc 1999; 50 (6): 762-7.

8. Pohle T, Helleberg M, Menzel J, Diallo R, Vestring T, Senninger N, et al. An extraordinary Dieulafoy's lesion presenting as varices of the gastric fundus. Gastrointest Endosc 2001; 54 (6): 776-9.

9. Fockens P, Meenan J, van Dullemen HM, Bolwerk CJ, Tytgat GN Dieulafoy's disease: endosonographic detection and endosonography-guided treatment. Gastrointest Endosc 1996; 44 (4): 437-42.

10. Nesje LB, Skarstein A, Matre K, Myking AO, Odegaard S. Dieulafoy's vascular malformation: role of endoscopic ultrasonography in therapeutic decision-making. Scand J Gastroenterol 1998; 33 (1): 104-8.

11. Chung IK, Kim EJ, Lee MS, Kim HS, Park SH, Lee MH, et al. Bleeding Dieulafoy's lesions and the choice of endoscopic method: comparing the hemostatic efficacy of mechanical and injection methods. 
Gastrointest Endosc 2000; 52 (6): 721-4.

12. Matsui S, Kamisako T, Kudo M, Inoue R. Endoscopic band ligation for control of nonvariceal upper GI hemorrhage: comparison with bipolar electrocoagulation. Gastrointest Endosc 2002; 55 (2): 214-8.

13. Nikolaidis N, Zezos P, Giouleme O, Budas K, Marakis G, Paroutoglou $\mathrm{G}$, et al. Endoscopic band ligation of Dieulafoy-like lesions in the upper gastrointestinal tract. Endoscopy 2001; 33 (9): 754-60.

14. Lee YJ, Oh JM, Park SE, Park JH. Successful treatment of a gastric
Dieulafoy's lesion with a hemoclip in a newborn infant. Gastrointest Endosc 2003; 57 (3): 435-6.

15. Folvik G, Nesje LB, Berstad A, Odegaard S. Endosonography-guided endoscopic band ligation of Dieulafoy's malformation: a case report. Endoscopy 2001; 33 (7): 636-8.

16. Ribeiro A, Vázquez-Sequeiros E, Wiersema MJ. Doppler EUS-guided treatment of gastric Dieulafoy's lesion. Gastrointest Endosc 2001; 53 (7): 807-9.

\title{
Enfermedad de Dieulafoy recidivante tratada quirúrgicamente: diagnóstico mediante ultrasonografía endoscópica
}

\author{
D. Martínez Ares, J. Souto Ruzo, J. Yáñez López, P. Alonso Aguirre, C. Gómez Mata², L. Valbuena \\ Ruvira ${ }^{1}$ y J. L. Vázquez Iglesias
}

Servicios de Aparato Digestivo, ${ }^{1}$ Anatomía Patológica $y^{2}$ Cirugía General. Complejo Hospitalario Universitario Juan Canalejo. A Coruña

\section{RESUMEN}

La enfermedad de Dieulafoy constituye una causa rara pero potencialmente muy grave de hemorragia digestiva. Se debe a la presencia de un vaso arterial de gran calibre en la submucosa, en contacto con una mucosa a la que debilita y erosiona, lo que puede conducir a un sangrado digestivo de gran magnitud. El diagnóstico endoscópico no siempre es fácil, siendo la ecoendoscopia una técnica que puede venir a solucionar estas dificultades. Además, dado que permite localizar con gran exactitud este vaso submucoso, puede contribuir a aumentar la precisión del tratamiento endoscópico mediante métodos mecánicos (hemoclips o bandas elásticas) o la combinación de métodos térmicos y la inyección de sustancias esclerosantes.

Presentamos a continuación un caso que ilustra perfectamente esta aplicación de la ultrasonografía endoscópica, definiendo perfectamente el sustrato morfológico de esta entidad.

Palabras clave: Enfermedad de Dieulafoy. Ultrasonografía endoscópica. Tratamiento quirúrgico. Tratamiento endoscópico.

\section{INTRODUCCIÓN}

La enfermedad de Dieulafoy representa una causa poco frecuente de hemorragia digestiva (1) que representa aproximadamente el $1 \%$ de las hemorragias digestivas altas no varicosas (2), cuyo grupo mayoritario, como es bien conocido, lo representa la úlcera péptica (3). No obstante, su incidencia real no es conocida con exactitud ya que algunos casos pueden no ser correctamente diagnosticados (4). La mayoría de estas lesiones suelen localizarse en la cavidad gástrica (1), aunque pueden aparecer en otras localizaciones del tubo digestivo como duodeno, yeyuno o incluso el recto. Esta entidad es poco frecuente pero que puede alcanzar elevadas cotas de gravedad, e incluso puede tener un desenlace fatal.

Se denomina también "persistencia del calibre arterial", caracterizándose por la presencia de una arteria de grueso calibre que atraviesa la submucosa, discurriendo 
en contacto con la mucosa (5). Mínimas roturas de esta mucosa (6), que generalmente se encuentra atrofiada, favorece la rotura del vaso y la subsiguiente hemorragia. Estas hemorragias pueden ser únicas o recidivantes. El diagnóstico es muy complicado si no se objetiva hemorragia activa, ya que puede no verse una lesión evidente en la mucosa (5). Inicialmente suele realizarse un tratamiento endoscópico con esclerosis asociada o no a coagulación con métodos térmicos o tratamiento mediante métodos mecánicos (hemoclips o bandas elásticas). Cuando no se consigue controlar de este modo la hemorragia o esta recidiva, suele indicarse la cirugía con resección del segmento afecto.

\section{CASO CLÍNICO}

Se trata de un varón de 39 años con dos ingresos previos, nueve y cuatro años antes, por HDA secundaria a enfermedad de Dieulafoy, uno de los cuales había requerido intervención quirúrgica. No refería otros antecedentes personales de interés, negaba ingesta etílica, tabaquismo u otros hábitos tóxicos. Negaba asimismo consumo de AINE y de cualquier otro fármaco.

En esta ocasión había consultado por un cuadro de 3 días de evolución caracterizado por un síndrome anémico intenso acompañado de deposiciones de carácter melénico. La analítica reflejaba una anemia severa con un hematocrito del $25 \%(38-50)$ y una hemoglobina de $7,5 \mathrm{~g} / \mathrm{dl}(13,5-18)$. En la endoscopia alta urgente se objetivó una lesión protuyente con mucosa conservada en la unión de cuerpo y fundus, sin sangrado activo en ese momento y que parecía corresponder a una enfermedad de Dieulafoy (Fig. 1). En la ecoendoscopia se describió una estructura tubular hipoecogénica, que correspondía al vaso arterial con calibre anormalmente grande en contacto con la mucosa, ulcerado en el vértice, sugestiva de enfermedad de Dieulafoy (Fig. 2). Se realizó escleroterapia endoscópica y fueron colocados dos hemoclips sobre la lesión. El paciente presentó en los días posteriores una recidiva hemorrágica, con hematemesis y rectorragias, presentando inestabilidad hemodinámica y requiriendo transfusión de 6 concentrados de hematíes. Por este motivo se decidió realizar un tratamiento quirúrgico. La intervención consistió en una gastrectomía parcial, y en el estudio anatomopatológico se objetivó un vaso aneurismático submucoso con erosión de la mucosa suprayacente, trombosis de la luz y zonas de hemorragia periférica (Fig. 3). La evolución posterior del enfermo fue satisfactoria, sin evidencia de recidiva hemorrágica.

\section{DISCUSIÓN}

El diagnóstico de la enfermedad de Dieulafoy no siempre es fácil, especialmente cuando durante la endoscopia no se aprecia sangrado activo, y puede requerirse la repetición de la exploración. Norton y cols. publicaron en 1999 una serie de 90 casos en las que se precisaron una media de 1,9 exploraciones para alcanzar el diagnóstico (7). En nuestro caso se presentó bajo la forma de una lesión con cierto aspecto nodular y con sangrado activo, todo ello muy sugestivo de una lesión de Dieulafoy, pero esta entidad puede presentar un aspecto que le haga pasar totalmente despercibido si no hay hemorragia activa o adoptar un aspecto tan abigarrado que simule el de las varices gástricas (8). Cuando existen dudas para el diagnóstico la ultrasonografía endoscópica y el doppler pueden ser de gran ayuda para localizar el vaso arterial de gran calibre en contacto con la mucosa que define esta lesión $(9,10)$; este hecho queda perfectamente ilustrado con las espectaculares imágenes ofrecidas por la ecoendoscopia en el caso que presentamos.

El tratamiento inicial de la enfermedad de Dieulafoy debe ser endoscópico. La hemostasia puede lograrse con similar eficacia con métodos mecánicos y con métodos térmicos combinados o no con la inyección de sustancias esclerosantes (1). De lo que no existe duda alguna en este momento es de que la esclerosis endoscópica como única actitud terapéutica no es aceptable, ya que su eficacia es claramente inferior a la combinación de esclerosis y métodos térmicos (2), y de los tratamientos mecánicos (11). En relación con estos últimos, tanto la colocación de bandas elásticas $(12,13)$, como la colocación de hemoclips $(11,14)$, se han mostrado como técnicas seguras, muy eficaces y con escasos costes. Estas técnicas pueden apoyarse en el doppler y en la ultrasonografía endoscópica para la localización del vaso con mayor precisión y de esta forma mejorar los resultados, especialmente en los casos en los que la lesión es poco evidente $(15,16)$. En nuestro caso, la ultrasonografía endoscópica nos confirmó el diagnóstico y guió la colocación de dos hemoclips. A pesar de que su colocación aparentemente fue precisa y de que también se había realizado esclerosis endoscópica sobre la lesión, se produjo una recidiva hemorrágica, motivo por el que se optó por la cirugía como solución definitiva.

En definitiva, la enfermedad de Dieulafoy es una entidad poco frecuente, en la que el diagnóstico no siempre es fácil, y en la que la ultrasonografía endoscópica puede ser útil tanto en el diagnóstico como para guiar una más precisa terapéutica endoscópica. Cuando esta fracasa o existe una recidiva hemorrágica el tratamiento definitivo suele ser el quirúrgico. 